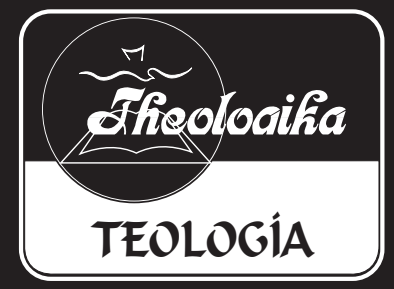

\title{
El uso del Antiguo Testamento en el Nuevo: Diagnóstico y prescripción sobre el debate actual
}




\section{Roy Graf}

Universidad Peruana Unión 


\section{RESUMEN}

“El uso del Antiguo Testamento en el Nuevo: Diagnóstico y prescripción sobre el debate actual" - El uso del Antiguo Testamento en el Nuevo ha sido objeto de estudio y debate debido a las implicancias hermenéuticas que dicho uso reviste para el intérprete contemporáneo. Un grupo de eruditos bíblicos sostiene que el Nuevo Testamento hace referencia al Antiguo siempre en un sentido literal, llano o atenido al contexto original. Otros en cambio, sostienen que el Nuevo Testamento hace uso de textos del Antiguo reinterpretándolos en un sentido diferente a su intención original; se asume que éstos son sacados fuera de su contexto o se les atribuye un significado adicional que no es evidente en los textos mismos. En este debate juega un papel importante el concepto de sensus plenior, que ha sido interpretado de diversas formas. El presente artículo hace un "diagnóstico" del estado actual del debate en sus diversos matices a través de una revisión de la literatura en relación al mismo, a la vez que propone algunas "prescripciones" que puedan conducir la discusión por una senda más productiva.

\section{SUMMARY}

"The use of the Old Testament in the New: Diagnosis and Prescription about the Actual Debate" - The use of the Old Testament in the New has been one subject of study and debate due the to hermeneutical implications that such use has for the comtemporary interpreter. Group of biblical scholars argue that the New Testament makes reference to the Old always in a literal or plain manner or adhere to the original context sense. However another hold the view that the New Testament uses the texts of Old Testament reinterpreting them in way contrary to its original intentions; it is assumed that these meanings are taken out of context or attribute to an additional meaning that is not evident in the texts themselves. In this debate, an article renewing the concept of sensus plenior, which has been interpreted in various forms, will play a very important role. This article makes a "diagnosis" of the current state of debate in its varied lights through a literature review while proposing some "prescriptions" that have the power to lead the discussion down a more productive path. 


\section{EL USO DEL ANTIGUO TESTAMENTO EN EL NUEVO: DIAGNÓSTICO Y PRESCRIPCIÓN SOBRE EL DEBATE ACTUAL}

\section{Introducción}

El uso del Antiguo Testamento (AT) en el Nuevo Testamento (NT) ha sido objeto de un extenso debate en buena medida por causa de las implicancias hermenéuticas del mismo. ${ }^{1}$ Existen más de trescientas

${ }^{1}$ Kaiser afirma que "la relación entre el AT y el NT permanece como uno de los máximos problemas, sino el principal, en la investigación bíblica de este siglo [veinte]". Walter C. Kaiser, Jr. The Uses of the Old Testament in the New (Chicago: Moody Press, 1985), 1. Davidson, más específicamente sostiene que "uno de los asuntos más cruciales involucrados en la inspiración de la Santa Escritura es la cuestión de la relación entre el Antiguo y el Nuevo Testamentos y en particular el uso de citas del AT por los escritores del NT". Richard M. Davidson, "New Testament Use of the Old Testament", Journal of the Adventist Theological Society 5:1 (1994) 14. Para una síntesis de los principales actores y puntos de vista involucrados en el debate sobre el uso del AT en el NT véase Darrell L. Bock, "Evangelicals and the Use of the Old Testament in the New: Part 1", Bibliotheca Sacra 142 (Julio 1985) 209-23; Darrell L. Bock, “Evangelicals and the Use of the Old Testament in the New: Part 2", Bibliotheca Sacra 142 (Octubre 1985) 302-19; Robert L. Thomas, "The New Testament Use of the Old Testament", The Master's Seminary Journal 13:1 (2002) 88-98.

Incluso en el marco de los círculos académicos adventistas han existido diferentes puntos de vista sobre el tema. Véase Davidson, "New Testament Use of the Old Testament", 15, y compárese con los siguientes: Gerhard Pfandl, "Ellen G. White and Hermeneutics”, en George W. Reid, ed., Understanding Scripture. An Adventist Approach (Silver Spring MD: Biblical 
cincuenta citas del AT en el NT sin contar las alusiones, paralelos verbales, ecos, etc. ${ }^{2}$ En cuanto a este fenómeno, algunos eruditos sostienen que el NT hace un uso de las citas, referencias o alusiones del AT siempre en un sentido literal, llano o ateniéndose al contexto. ${ }^{3}$ Las declaraciones del AT estarían siempre interpretadas teniendo en cuenta su contexto original y sin pretender leer en él algo nuevo o reinterpretarlo a la luz de una nueva agenda teológica. ${ }^{4}$ Esto se aplicaría también a aquellos pasajes del AT a los que el Nuevo les atribuye un carácter predicativo y que son interpretados como encontrando cumplimiento en hechos del NT. Según

Research Institute General Conference of Seventh-day Adventist, 2005), 314, 315; Raymond F. Cottrel, "Ellen G. White's Evaluation and Use of the Bible", en Gordon M. Hyde, ed., A Symposium on Biblical Hermeneutics (Washington: Biblical Research Committee, General conference of Seventh-Day Adventist, 1974), 161; Herbert E. Douglass, Mensajera del Señor: El ministerio profético de Elena G. de White, trad. Tulio N. Peverini (Buenos Aires: Asociación Casa Editora Sudamericana, 2000), $224 n 37$.

${ }^{2}$ Véase Kurt Aland, et al. eds., The Greek New Testament, 3ra ed. (Nueva York: Sociedades Bíblicas Unidas, 1975), 897-911.

${ }^{3}$ Véase Davidson, "New Testament Use", 34, donde el autor concluye que "los escritores del NT consistentemente permanecen fieles a los pasajes originales en sus contextos inmediatos y más amplios".

${ }^{4}$ Beale está convencido de que "una vez que se consideran las presuposiciones hermenéuticas y teológicas de los escritores del Nuevo Testamento, no hay ejemplos claros donde ellos hayan desarrollado un sentido a partir del Antiguo Testamento que sea inconsistente o contradictorio con algún aspecto de la intención original del Antiguo Testamento". G. K. Beale, "Positive Answer to the Question Did Jesus and His Followers Preach the Right Doctrine from the Wrong Texts? An Examination of the Presuppositions of Jesus 'Jesus and the Apostles' Exegetical Method", en G. K. Beale, ed., The Right Docrine from the Wrong Texts? Essays on the Use of the Old Testament in the New (Grand Rapids, MI: Baker Books, 1994), 398. 
este punto de vista los autores del AT comprendían todas las implicancias de sus predicciones proféticas. ${ }^{5}$

Por otro lado, existe una corriente en sentido más o menos opuesto que cree que los autores del NT a menudo han utilizado citas o referencias a pasajes del AT reinterpretándolos en un sentido diferente a su intención original o sacándolos aparentemente fuera de su contexto. ${ }^{6}$ Este punto de vista sostiene también que frecuentemente se toman pasajes del AT a los cuales se les atribuye un

${ }^{5}$ Kaiser dice al respecto que "los escritores [bíblicos] pueden y deben tener una adecuada comprensión del asunto sobre el cual ellos escriben incluso si no tienen un control total de todos los detalles y partes que pertenecen al asunto". Kaiser, Jr., The uses of the Old Testament in the New, 21. La cursiva es del original.

${ }^{6}$ Ver Richard N. Longenecker, "Negative Answer to the Question 'Who is the Prophet Talking About?' Some Reflections on the New Testament's Use of the Old", en The Right Docrine from the Wrong Texts?, 377, donde afirma que "hay veces cuando el Nuevo Testamento cita el Antiguo Testamento en formas que parecen muy fuera de contexto aunque pretende un cumplimiento en Cristo o en la experiencia cristiana para estos pasajes". Al referirse a la cita de Os 11:1 por Mt 2:15 sugiere que el evangelista está "releyendo su Antiguo Testamento desde una perspectiva escatológicamente realizada y cristológica", ibid. Véase también Richard N. Longenecker, Biblical Exegesis in the Apostolic Period, 2da ed. (Grand Rapid, MI: Eerdmands Publishing Company, 1999), xxxiii. Para él, hay instancias en el NT "donde la Escritura es citada de una manera que va más allá de su sentido literal, o más allá de lo que se puede determinar por las reglas de la exégesis histórico-crítica que haya sido el mensaje del autor del Antiguo Testamento para su tiempo". Thomas parece acordar con Longenecker al proponer varios ejemplos de citas del AT tomadas fuera de contexto en el NT: "En tales casos los escritores del NT tomaron palabras del AT y las aplicaron a situaciones enteramente diferentes de aquellas que fueron previstas en los contextos correspondientes del AT. Ellos desatendieron la dirección principal de los significados histórico-gramaticales de los pasajes del AT y los aplicaron en forma diferente para adaptarlos a los distintos asuntos que estaban comunicando"; Thomas, "The New Testament Use of the Old Testament", 83. 
sentido profético en el NT, que iría más allá de su intención original. ${ }^{7}$ Este sentido adicional del texto, que no es evidente en el texto del AT, es a menudo denominado sensus plenior. ${ }^{8}$ Se suele afirmar también que los autores del NT, por ser inspirados, estarían capacitados para encontrar en los pasajes del AT un sentido que no era obvio para los autores y lectores del $\mathrm{AT}^{9}$ pero que estaba previsto en el marco de la revelación progresiva de Dios al hombre.

\section{Diagnóstico sobre la situación actual del debate}

Esta discusión asume matices diversos en la literatura reciente sobre el tema. En un extremo del espectro, Walter Kaiser Jr. niega la posibilidad de un

${ }^{7}$ Longenecker cita varios ejemplos bíblicos: “los más obvios son las diez u once citas de 'fórmula de cumplimiento' del Evangelio de Mateo, la aplicación de Sal 16:8-11 a la resurrección del Mesías en Hechos 2:25-28, el uso de Sal 2:1-2 en la alabanza a Dios en Hechos 4:25-26, y la cita de Sal 68:18 en una manera que revierte enteramente su significado original en Ef 4:8"; Longenecker, xxxiii.

${ }^{8}$ Es decir, "sentido completo". Véase por ejemplo, Longenecker, Biblical Exegesis in the Apostolic Period, xxxi-xxxiv. Para una síntesis histórica del debate en relación al sensus plenior véase Henning Graf Reventlow, Problems of Biblical Theology in the Twentieth Century, John Bowden trad. (Filadelfia: Fortress Press, 1986), 37-47.

9Thomas, "The New Testament Use of the Old Testament": 87: Los escritores del NT poseían el don del apostolado y/o el don de profecía que los capacitaba para recibir y transmitir a otros revelaciones directas de Dios ... Estos dones capacitaban a quienes los recibían para practicar lo que se llama 'exégesis carismática' en el uso de los textos del AT. La práctica comportaba el hallazgo de significados escondidos o simbólicos que se podían revelar a través de un intérprete que poseyera revelación divina". 
sensus plenior. Para él un pasaje del AT, no puede tener un doble sentido o significado, ni tampoco un doble cumplimiento, por ejemplo literal y espiritual. ${ }^{10}$ Kaiser abraza la idea de que "el axioma de interpretación que hace posible la comunicación es que 'ningún pasaje en ninguna literatura tiene más que un sentido' a menos que la literatura nos señale que estamos tratando con frases con doble sentido, enigmas, o cosas por el estilo".${ }^{11}$ De lo contrario, la revelación no sería posible. En el caso de los textos proféticos del AT, Kaiser aboga por lo que llama "promesa genérica". La profecía del AT no anunciaría solamente un cumplimiento culminante sino una serie de eventos que participan y se dirigen hacia un evento culminante, pero donde todos son vistos como uno. ${ }^{12}$ Se trataría de un cumplimiento genérico y no doble o múltiple. Bajo estas premisas, sólo los textos relacionados precedentes a una profecía podrían ayudar en su interpretación, y no los posteriores, descartando así que el NT deba ser utilizado para interpretar el AT. La intención de un texto sería siempre singular y no doble o múltiple en sentido, ${ }^{13}$ preservando así la unidad entre la intención divina y la humana en cuanto al significado de un texto.

Del mismo modo, Robert Thomas concuerda en que un texto sólo puede tener un significado. ${ }^{14} \mathrm{Sin}$

${ }^{10}$ Kaiser, Jr. The Uses of the Old Testament in the New, 63, 64.

${ }^{11}$ Ibíd., 64.

${ }^{12} I b i ́ d ., 67$.

${ }^{13}$ Ibíd., 68-71.

${ }^{14}$ Véase Robert L. Thomas, "The principle of single meaning", The Master's Seminary Journal 12:1 (2001) 33-47. 
embargo, para él ese único significado no descarta la posibilidad de que un autor inspirado del NT establezca una aplicación inspirada no literal de un pasaje del NT, que va más allá de su sentido original. A este fenómeno Thomas se refiere como "aplicación inspirada del sensus plenior". ${ }^{15}$ Los intérpretes contemporáneos no podrían utilizar este recurso interpretativo, sin embargo, por el simple hecho de no tener la asistencia de la inspiración. ${ }^{16}$ Thomas reconoce que su punto de vista es muy cercano al del reconocido erudito en NT John Walton, más allá de algunas diferencias terminológicas. ${ }^{17}$

Gregory Beale, sin embargo, no está de acuerdo con que no se puede reproducir la exégesis del NT. Para él la exégesis de los escritores del NT es "contextual", es decir, atenida a su contexto original. Sigue a Charles Dodd al afirmar que los autores del NT tenían en cuenta un contexto más amplio de los versículos que citan del $\mathrm{AT}^{18}{ }^{18}$ lo que podría explicar por qué a menudo dan la impresión de ignorar el contexto inmediato. Reconoce que los autores del NT "despliegan una variedad de grados de conciencia del contexto literario, así como

${ }^{15}$ Véase su discusión en Thomas, “The New Testament Use of the Old Testament": 79, 80. Thomas reconoce, sin embargo, que no todos los textos del AT que hallan cumplimiento en el NT son interpretados de manera no literal por los autores del NT. De hecho él provee una extensa revisión de pasajes del AT que tienen un cumplimiento literal en el NT. Véase ibid., 80-82.

${ }^{16}$ Ibíd., 86, 87.

${ }^{17}$ Ibíd., 94, 97. Véase el artículo de John H. Walton, "Inspired Subjectivity and Hermeneutical Objectivity", The Master's Seminary Journal 13:1 (2002): 65-77.

${ }^{18}$ Beale, "Positive Answer", 390. 
también quizá del contexto histórico" ${ }^{19}$ Pero creeque "las partes más recientes de la historia bíblica [NT] funcionan como el contexto más amplio para interpretar las partes anteriores [AT] porque todas ellas tienenel mismo, último autor divino quien inspira a los diferentes autores" ${ }^{20}$ De esta premisa deduce que "Cristo como el centro de la historia es la clave para interpretar las porciones anteriores del Antiguo Testamento y sus promesas". ${ }^{21}$ Se puede percibir que en este punto Beale está en total contraposición con Kaiser para quien sólo la revelación anterior de un texto sirve como contexto para su interpretación. Para Beale es todavía más importante la revelación posterior. Asume un punto de vista moderado sobre la existencia de un sensus plenior. Los autores del NT expanden las declaraciones del AT pero de una forma que "no contraviene la integridad de los textos anteriores sino que los desarrolla en una forma que es consistente con la comprensión del autor del Antiguo Testamento de la forma en la cual Dios interactúa con su pueblo -lo cual es el factor unificador entre los Testamentos". ${ }^{22}$ Beale concluye que los intérpretes contemporáneos pueden reproducir el método interpretativo del NT aunque "no podamos tener la misma certeza epistemológica acerca de nuestras conclusiones interpretativas" 23 debido a la ausencia del don de la inspiración.

\footnotetext{
${ }^{19}$ Beale, “Positive Answer", 391.

${ }^{20}$ Ibid., 392.

${ }^{21}$ Ibíd. La cursiva es del original.

${ }^{22}$ Ibíd., 393.

${ }^{23}$ Ibíd., 399.
} 
El interés de Beale por la discusión acerca del uso del AT en el NT se ve reflejado en la publicación del libro The Right Doctrine from the Wrong Texts?, en el que recopila artículos y ensayos de diversos eruditos en relación al tema en cuestión que expresan opiniones contrapuestas en aspectos como la fidelidad de los autores del NT a la intención de los autores del AT (parte 2), su respeto por el contexto (partes 3, 4 y 5), el uso de la tipología en el NT (parte 6) y la utilidad de los métodos exegéticos del NT para el intérprete contemporáneo (parte 7). ${ }^{24}$

${ }^{24}$ Esta obra agrupa ensayos de autores tan diversos como Kaiser, Jr., R. T. France, C. H. Dodd, David L. Baker, Richard N. Longenecker y el propio Beale. Cabe destacar que Beale no está solo en sus puntos de vista. Anteriormente Hasel, por ejemplo, ha afirmado que "El 'sentido completo' [sensus plenior] de la revelación bíblica es provisto por una revelación posterior a los profetas antiguos. La inspiración es una guía segura para obtener el sentido completo de cualquier parte de las Escrituras. Gerhard Hasel, La interpretación de la Biblia, trad. Sylvia Socoloske de González (Buenos Aires: Ediciones SALT, 1986), 132. Hasel agrega que "no se lee un significado nuevo en la profecía anterior, pero [sic] a través de la inspiración el 'sentido completo' o significado más amplio, es revelado sin ninguna mala aplicación o reinterpretación donde un significado ajeno se sobrepone al original. Es la intención divina que el 'sentido completo' genuino y normativo fuera revelado a través de la inspiración divina en un momento posterior. Este procedimiento resguarda a las Escrituras de interpretaciones subjetivas y personales y asegura la interpretación propia de la Biblia basada en el principio de la analogía de la fe", ibíd., 133. Beale ha sido coeditor junto a D. A. Carson del recientemente publicado Commentary on the New Testament Use of the Old Testament (Grand Rapids, MI: Baker Academic, 2007). Esta obra comenta todas las citas que el NT hace del AT y las probables alusiones. Sin embargo, no se interna en el debate hermenéutico (ver xxiii), aunque parece evidente que los colaboradores de la obra parten de presuposiciones hermenéuticas conservadoras. Otros autores que podrían colocarse en la misma línea de Beale son Donald A. Hagner, "The Old Testament in the New Testament", en Samuel J. Schultz y Morris A. Inch, eds., Interpreting the Word of God. Festschrift in Honor of Steven Barabas (Chicago: Moody Press, 1976), 78-104 (ver especialmente 
La postura de Beale sobre la forma en que el NT utiliza al AT ha sido expresada también en el marco de polémicas con otros eruditos bíblicos tales como Peter Enns ${ }^{25}$ y Richard Longenecker quienes sostienen que el NT interpreta pasajes del AT sin tener en cuenta su contexto. Longenecker, uno de los eruditos más influyentes sobre este tema, ha asignado un papel destacado al impacto de la hermenéutica judía del primer siglo sobre los escritores del NT. ${ }^{26}$ Aunque él reconoce "la naturaleza cristocéntrica y la cualidad neumática" de la exégesis del $\mathrm{NT},{ }^{27}$ piensa que la exégesis que hace el NT del AT estuvo influida por la exégesis judía que apelaba a recursos tales como la interpretación de tipo midrash, pesher y la interpretación alegórica, además de la interpretación literal. Esto lo lleva a asumir que el sensus plenior no es solamente una extensión de un sentido ya implícito en el texto. Involucra que los autores del NT citen a menudo a los del AT sin dar importancia al contexto. Para ello estarían autorizados, sin embargo,

90-104). Véase también Hans K. LaRondelle, The Israel of God in Prophecy. Principles of Prophetic Interpretation (Berrien Spring, MI: Andrews University Press), especialmente los capítulos 5 (60-80) y 7 (98-123).

${ }^{25}$ Véase la secuela de artículos de este debate: G. K. Beale, "Did Jesus and the Apostles Preach the Right Doctrine from the Wrong Texts? Revisiting the Debate Seventeen Years Later in the Light of Peter Enns' Book, Inspiration and Incarnation", Themelios 32:1 (2006?) 18-43; Peter Enns, "Response to Professor Greg Beale", Themelios 32:3 (2006?) 5-13; G. K. Beale, "A Surrejoinder to Peter Enns", Themelios 32:3 (2006?) 14-25.

${ }^{26}$ Sobre la relación entre exégesis judía y cristiana véase también Craig A. Evans y William F. Stinespring, eds., Early Jewish and Christian Exegesis. Studies in Memory of William Hugh Brownlee (Atlanta: Scholars Press, 1987).

${ }^{27}$ Longenecker, Biblical Exegesis in the Apostolic Period, xxx. 
por el hecho de ser inspirados. Por lo tanto, Longenecker afirma que los intérpretes contemporáneos no deben reproducir la exégesis del NT (cuando ésta se aleja de la senda de la literalidad). ${ }^{28}$ Ecos de estos puntos de vista se pueden encontrar también en otros eruditos tales como Earle Ellis ${ }^{29}$ o Steve Moyise. ${ }^{30}$ Moyise ha sostenido que "las citas escriturales en el Nuevo Testamento no siempre evocan las estructuras completas de significado [de los textos del AT y sus respectivos contextos] y esto no debería, por lo tanto, ser asumido como una presuposición". ${ }^{31}$ En otras palabras, para Moyise, las citas del AT en el NT no siempre se hacen desde la perspectiva de un contexto amplio del AT como Beale y otros han sostenido. ${ }^{32}$ Esto lo lleva a afirmar que, en

${ }^{28}$ Para bibliografía adicional a la ya citada de este autor véase Richard N. Longenecker, "Prolegomena to Paul's Use of Scripture in Romans", Bulletin for Biblical Research 7 (1997) 145-168; "Can We Reproduce the Exegesis of the New Testament?", Tyndale Bulletin 21 (1970) 3-38.

${ }^{29}$ E. Earle Ellis, "How Jesus Interpreted His Bible", Criswell Theological Review 3:2 (1989) 341-351; “Jesus' Use of the Old Testament and the Genesis of New Testament Theology", Bulletin for Biblical Research 3 (1993): 59-75.

${ }^{30}$ Steve Moyise, "Quotations", en S. E. Porter y C. D. Stanley, eds., As it is Written. Studying Paul's Use of Scripture (Atlanta: Society of Biblical Literature, 2008), 15-28; "Scripture in the New Testament: Literary and Theological Perspectives", Neotestamentica 42:2 (2008): 305-326; "Respect for Context Once More", Irish Biblical Studies 27 (2006): 24-31.

${ }^{31}$ Moyise, "Scripture in the New Testament: Literary and Theological Perspectives", 324.

${ }^{32}$ Véase pp. 5-6 de este trabajo. Véase también Steve Moyise, "Seeing the Old Testament Through a Lens", Irish Biblical Studies 23 (Jan 2001) 36-42, donde Moyise reacciona a la postura de Beale sobre cómo el Apocalipsis usa el AT. 
lo que respecta a la interpretación de las Escrituras por parte de los intérpretes modernos, "nosotros no podemos retornar a la exégesis pre-crítica más de lo que Pablo podía retornar a la interpretación farisaica. Una interpretación trinitaria no abandonará el pasado pero tampoco vivirá en el pasado". ${ }^{33}$

En un intento por conciliar diferencias entre autores como Longenecker y Beale, Dan McCartney ha propuesto lo que él considera una tercera posibilidad: "Deseo sugerir una tercera respuesta: Los escritores del NT no estaban haciendo exégesis histórico-gramatical ni interpretaron consistentemente de acuerdo a los significados del contexto histórico, pero nosotros deberíamos seguir su ejemplo exegético de todos modos". ${ }^{34}$ Para McCartney, tanto Longenecker como Beale "simplemente asumen que el método exegético histórico gramatical es el correcto y es la única manera correcta de emprender la tarea de interpretación" ${ }^{35}$ Longenecker piensa que los escritores del NT no usaron ese método por estar condiciondos por la hermenéutica judía, pero que los intérpretes contemporáneos lo debieran seguir, en tanto que Beale sostiene que los

${ }^{33}$ Steve Moyise, "Can We Use the New Testament in the Way which the New Testament Authors use the Old Testament?", In die Skriflig 36:4 (2002) 659.

${ }^{34}$ Dan G. McCartney. "Should we Employ the Hermeneutics of the New Testament Writers?" (ensayo presentado como ponencia en la 55th Annual Meeting of the Evangelical Theological Society, 19-21, Atlanta, GA, November 2003), 1.

${ }^{35} I b i ́ d ., 6$. La cursiva es del original. McCartney señala que incluso Walton y Kaiser también asumen que el método histórico-gramatical es el único correcto. 
apóstoles sí usaron el método histórico gramatical y que éste debe ser el ejemplo a seguir por el intérprete actual. Por lo tanto, sostiene McCartney, "lo que vincula a todos estos puntos de vista, liberal y conservador, es la simple suposición de que sólo la exégesis histórico-gramatical es legítima para el intérprete cristiano del presente". ${ }^{36}$ Sin embargo, la clave para entender la forma en que los escritores del NT interpretan los textos del AT es que su entendimiento está "conectado a lo que Dios ha revelado subsecuentemente, y particularmente a lo que Jesús y Pablo dicen acerca del AT" ${ }^{37}$ McCartney concluye que:

debemos . . como Jesús y los apóstoles, continuar leyendo el AT en el contexto no sólo de la Biblia como un todo, sino en el contexto de la historia redentiva como un todo. En particular debemos leer el AT con ojos cristianos, con los ojos del que cree en el AT como una parte de un libro evangélico, como una historia vital que llega ser nuestra historia debido a que ésta es la historia de Cristo. ¿Deberíamos emplear la hermenéutica de los escritores del NT? En verdad, debemos. ${ }^{38}$

El propio McCartney reconoce, sin embargo, que su postura cristológica es un tipo de exégesis que no ofrece "el control metodológico absoluto que la ciencia demanda". ${ }^{39}$ En cualquier caso su punto de

${ }^{36}$ McCartney. "Should we Employ the Hermeneutics of the New Testament Writers?", 7.

${ }^{37}$ Ibíd., 14.

${ }^{38}$ Ibíd., 17.

${ }^{39}$ Dan McCartney y Charles Clayton, Let the Reader Understand. A Guide to Interpreting and Applying the Bible (Wheaton, IL: Victor Books/SP 
vista tiene la ventaja de internarse en el terreno de las presuposiciones que los eruditos manejan en relación a la valoración hermenéutica que hacen del uso del AT en el Nuevo. Otros autores han seguido una línea similar a la de McCartney. Scott Swanson, por ejemplo, resume correctamente la naturaleza del debate dentro del ámbito evangélico al sostener que "el desacuerdo está entre aquellos que insisten en que el NT capta correctamente la intención del autor humano [del AT] estrictamente de acuerdo a la exégesis histórico-gramatical, y aquellos que ven al NT como identificando correctamente la intención del autor divino a la luz de la revelación adicional [sensus plenior]". ${ }^{40}$ AunqueSwanson concuerda con Kaiser en que se ha subestimado la comprensión de los escritores del AT de sus declaraciones mesiánicas o escatológicas (es decir la comprensión del sensus plenior), sin embargo, las mismas no pueden entenderse plenamente a partir de una exégesis histórico gramatical y sin la asistencia del NT. ${ }^{41}$ Swanson es más explícito que McCartney al afirmar que "el significado que el NT encuentra en el AT puede ir más allá de la perspectiva inmediata del autor humano", pero este significado "es una implicación legítima o extensión de las palabras y el significado intencional del autor humano". ${ }^{42}$

Publication, 1994), 162. Los autores añaden que el control metodológico "opera por un control 'de fe' que depende de la sensibilidad y sumisión hermenéutica a Dios para sus resultados", ibíd.

${ }^{40}$ Scott A. Swanson, "Can We Reproduce The Exegesis Of The New Testament? Why Are We Still Asking?”, Trinity Journal 17:1 (1996): 68-69.

${ }^{41}$ Ibíd., 69, 70.

${ }^{42}$ Ibíd., 69. 
Semejante diversidad de puntos de vista ha llevado a otros como Darrell Bock a asumir lo que él llama una "aproximación ecléctica" ${ }^{43}$ al problema. Bock espiga en el pensamiento de autores como Kaiser, Jr., S. Lewis Johnson, James I. Packer, Elliott E. Johnson, Ellis, Longenecker, Bruce Waltke y otros de los que trata de retener el "trigo" y dejar a un lado la "paja". ${ }^{44}$ Sin embargo, su postura ha sido criticada por Thomas quien señala que "el eclecticismo en la hermenéutica generalmente implica inconsistencia debido a que se siguen varias aproximaciones en conflicto". ${ }^{45}$

Unagrancantidad deestudiossobreelusodel ATenel NT han sido realizados desde las diferentes aproximaciones hermenéuticas descritas. De especial importancia han sido los estudios hechos sobre el abundante uso del AT por los autores de los Evangelios (especialmente, Mateo), ${ }^{46}$

${ }^{43}$ Bock, "Evangelicals and the Use of the Old Testament in the New: Part 1", 220.

${ }^{44}$ Bock "Evangelicals and the Use of the Old Testament in the New: Part 2", 316.

${ }^{45}$ Thomas, "The New Testament Use of the Old Testament", 92.

${ }^{46}$ Véase por ejemplo Maarten J. J. Menken, “The Old Testament Quotation in Matthew 27,9-10: Textual Form and Context", Biblica 83:3 (2002) 305-328. Lee Campbell, "Matthew's Use of the Old Testament: A Preliminary Analysis", Xenos Online Journal 3, http://www.xenos.org/ ministries/crossroads/OnlineJournal/issue3/mtmain.htm (consultado: 2 de febrero, 2009); Wim J. C. Weren, "The Use of Isaiah 5,1-7 in the Parable of the Tenants (Mark 12,1-12; Matthew 21,33-46)", Biblica 78:1 (1998) 1-26; Glenn Balfour, "The Jewishness of John's use of the Scriptures in John 6:31 y 7:37-38", Tyndale Bulletin 46:2 (1995) 357-380; Andrew Warren, "Did Moses permit divorce? Modal wěga ātal as Key to New Testament Readings of Deuteronomy 24:1-4", Tyndale Bulletin 49:1 (1998) 39-56; Steve Moyise, "The Wilderness Quotation in Mark 1.2-3", en R. S. Sugirtharajah, ed., 
incluyendo trabajos que tratan de extraer implicancias hermenéuticas de ese uso. ${ }^{47}$ También se han estudiado las referencias paulinas al AT particularmente en la epístola a los Romanos, ${ }^{48}$ la epístola a los Gálatas ${ }^{49}$ y la homilía a los

Wilderness. Essays in Honour of Frances Young (London \& New York: T\&T Clark, 2005), 78-87; Joel B. Green, "The Problem of a Beginning: Israel's Scriptures in Luke 1-2", Bulletin for Biblical Research 4 (1994) 61-86; Charles A. Kimball III, "Jesus'Exposition of Scripture in Luke 20:9-19: An Inquiry in Light of Jewish Hermeneutics", Bulletin for Biblical Research 3 (1993) 77-92; F. F. Bruce, "The Book of Zechariah and the Passion Narrative", Bulletin of the John Rylands Library 43 (March 1961) 336-353.

${ }^{47}$ Por ejemplo Craig Blomberg, "Interpreting Old Testament Prophetic Literature in Matthew: Double Fulfilment", Trinity Journal 23 (Spring 2002) 17-33. Bloomberg propone un doble cumplimiento de las profecías de Isaías, uno en tiempo del profeta y otro en tiempo del NT, lo que para él no implica necesariamente un sensus plenior, ya que el profeta sería consciente del cumplimiento inmediato y del cumplimiento escatológico de sus profecías.

${ }^{48}$ Véase por ejemplo Leslie C. Allen, "The Old Testament in Romans I-VIII", Vox Evangelica 3 (1964) 6-41; Steve Moyise, "Paul and Scripture in Dispute: Romans 2:24 as Test-Case", Proceedings of the Irish Biblical Association 29 (2006) 78-96; Steve Moyise, "The Catena of Romans 3:10-18", Expository Times 106 (1995) 367-370; Michael Cranford, “Abraham in Romans 4: The Father of All Who Believe", New Testament Studies 41:1 (1995) 71-88; John A. Battle, Jr., "Paul's Use of the Old Testament in Romans 9:25-26", Grace Theological Journal 2:1 (1981) 115-29.

${ }^{49}$ Timothy G. Gombis, "The 'Transgressor' and the 'Curse of the Law'. The Logic of Paul's Argument in Galatians 2-3", New Testament Studies 53 (2007) 81-93; Ardel Caneday, " "Redeemed from the Curse of the Law'. The Use of Deut 21:22-23 in Gal 3:13", Trinity Journal 10:2 (1989) 185-209; Joel Willitts, "Context Matters: "Paul' Use of Leviticus 18:5 in Galatians 3:12", Tyndale Bulletin 54:2 (2003) 105-122; Moisés Silva, "Abraham, Faith, and Works: Paul's Use of Scripture in Galatians 3:6-14", Westminster Theological Journal 63:2 (2001) 251-267; Lloyd Gaston, "Abraham and the Righteousness of God", Horizons in Biblical Theology 2:1(1980) 39-68; F. F. Bruce, "Abraham had two Sons: A Study in Pauline Hermeneutics", en Huber L. Drumwright y Curtis Vaughan, eds., New Testament Studies: Essays in Honor of Ray Summers in His Sixty-Fifth Year, (Waco, TX: Markham Press Fund, Baylor University Press, 1975), 71-84. 
Hebreos. ${ }^{50}$ De la misma manera se ha analizado la forma paulina de citar los Salmos ${ }^{51}$ y el libro de Isaías. ${ }^{52}$ También han sido objeto de estudio el uso del AT en Hechos, ${ }^{53}$ las epístolas universales ${ }^{54}$ y el Apocalipsis. ${ }^{55}$ Incluso se han realizado estudios comparativos de la hermenéutica de distintos libros del NT. ${ }^{56}$ Todos estos trabajos, sin

${ }^{50}$ Stephen Motyer, "The Psalm Quiotations of Hebrews 1: A Hermeneutic-Free Zone?", Tyndale Bulletin 50:1 (1999) 3-22; Tim Clark, "New Testament Use of the Old Testament: A Case Study from Hebrews 1-2", Xenos Online Journal 3, http://www.xenos.org/ministries/crossroads/ OnlineJournal/issue3/ntotherbr.htm (consultado: 2 de febrero, 2009); Peter E. Enns, "Creation and Re-Creation: Psalm 95 and Its Interpretation in Hebrews 3:1-4:13", Westminster Theological Journal 55:2 (1993) 255-280; John Proctor, "Judgement or Vidication? Deuteronomy 32 in Hebrews 10:30", Tyndale Bulletin 55:1 (2004) 65-80.

${ }^{51}$ Véase Allan M. Harmon, "Aspects of Paul's Use of the Psalms", Westminster Theological Journal 32:1 (1969) 1-23.

${ }^{52}$ Véase Douglas A. Oss, "A Note on Paul's Use of Isaiah", Bulletin for Biblical Research 2 (1992) 105-112.

${ }^{53}$ Véase por ejemplo Robert W. Wall, "The Function of LXX Habakkuk 1:5 in the Book of Acts", Bulletin for Biblical Research 10:2 (2000) 247-258.

${ }^{54}$ Véase por ejemplo Karen H. Jobes, "Got Milk? Septuagint Psalm 33 and the Interpretation of 1 Peter 2:1-3", Westminster Theological Journal 64:1 (2002) 1-14.

${ }^{55}$ Steve Moyise, "Singing the Song of Moses and the Lamb: John's Dialogical Use of Scripture, Andrews University Seminary Studies 42: 2 (2004) 347-360. Véase también Hans K. LaRondelle, Las profecías del fin. Enfoque contextual-bíblico, trad. David P. Gullón (Buenos Aires: Asociación Casa Editora Sudamericana, 1999).

${ }^{56}$ Véase por ejemplo, G. W. Grogan, "The New Testament Interpretation of the Old Testament: A Comparative Study", Tyndale Bulletin 18 (1967) 54-76, quien compara la hermenéutica de Mateo, Hebreos y Apocalipsis con la usada por Cristo mismo. Véase también O. Palmer Robertson, "Genesis 15:6: New Covenant Expositions of an Old Covenant Text", Westminster Theological Journal 42:2 (1980) 259-289. 
embargo, reflejan perspectivas hermenéuticas muy disímiles.

Este panorama de desacuerdo ha conducido a Dennis Stamps a afirmar que "el consenso es improbable sobre este asunto en la medida que haya tal diversidad de perspectivas y agendas teológicas. Quizá lo que uno puede esperar en relación a esto es que el estudio adicional será más preciso en definir términos y en declarar los intereses hermenéuticos y la perspectiva teológica informando un estudio en particular". ${ }^{57}$

En otras palabras, Stamps asume que un mayor estudio de este tema sólo producirá claridad en cuanto a las presuposiciones desde las cuales la investigación del uso del AT en el NT es abordado por cada erudito, pero no producirá mayor claridad sobre el tema en sí mismo.

\section{Prescripciones con miras al futuro}

El cuadro resultante del diagnóstico del debate actual, puede parecer francamente desalentador. ¿Es posible acaso arribar a un "puerto seguro" o conceso en relación a la comprensión del uso del AT en el Nuevo? Indudablemente, un debate de tal magnitud no puede ser resuelto en el marco de los estrechos límites de un artículo. Pero a partir de su análisis previo es posible elaborar algunas prescripciones con miras a conducir el debate por derroteros más productivos.

${ }^{57}$ Dennis L. Stamps, "The Use of the Old Testament in the New Testament as a Retorical Device: A Methodological Proposal", en Stanley E. Porter, ed., Hearing the Old Testament in the New Testament, (Grand Rapids, MI: Eerdmans, 2006), 23. 
Aunque no es necesario compartir el pesimismo de Stamps, es posible coincidir con él en que, en buena medida, la dificultad en el debate está relacionada con intereses hermenéuticos y teológicos opuestos. ${ }^{58}$ Y aunque no siempre es específicamente mencionado por los participantes en el debate, entre esos intereses teológicos figuran el de defender una determinada interpretación de la revelación y la inspiración. Avances recientes en la comprensión de la doctrina de la revelación-inspiración sugieren que tanto las interpretaciones tradicionales y conservadoras sobre la inspiración-inspiración, como las perspectivas modernas sobre la misma han construido extensamente sobre presuposicionesdeordenfilosóficoynobíblico. ${ }^{59}$ Estoimplica que se requiere que los estudios adicionales que se hagan sobre el tema deben permitir que la Biblia misma provea las presuposiciones teológicas y hermenéuticas necesarias para el abordaje apropiado de la comprensión del uso del AT en el Nuevo. Después de todo, "el procedimiento científico básico requiere que derivemos nuestras presuposiciones hermenéuticas del asunto que deseamos entender"; $; 0$ es decir, que nuestras presuposiciones hermenéuticas sobre como el AT es utilizado por el NT deben ser extraídas de la Biblia misma y no de fuentes ajenas a ella.

\section{${ }^{58}$ Ver Stamps, 23.}

${ }^{59}$ Véase al respecto Fernando L. Canale, Back to Revelation-Inspiration: Searching for the Cognitive Foundation of Christian Theology in a Postmodern World (Lanham, MD: University Press of America, 2001).

${ }^{60}$ Fernando Canale, "Revelation and Inspiration", en George W. Reid, ed., Understanding Scripture: An Adventist Approach (Silver Spring, MD: Biblical Research Institute, 2005), 62. 
En segundo lugar, y en relación con lo recién mencionado, es necesario tener en cuenta cuál fue la intención de los autores inspirados del NT al citar a sus pares del AT. Los autores del NT no necesariamente tuvieron la intención de establecer modelos precisos en cuanto a cómo hacer exégesis de textos inspirados. ${ }^{61}$ Esto implica que el mensaje que los autores buscaban transmitir puede ser más importante que el método a través del cual se transmite. Por otro lado, la "exégesis" de los autores del NT es de carácter más espontáneo, sin necesariamente seguir todos los pasos que actualmente seguiría un intérprete contemporáneo que está mucho más lejos de la cultura y los tiempos bíblicos.

En otra dirección, y en acuerdo con McCartney, no es posible aplicar lisa y llanamente el rótulo de método histórico-gramatical a la interpretación que el NT hace del Antiguo. ${ }^{62}$ La razón es que los autores del NT no estaban necesariamente interesados solamente en el sentido llano o "evidente" del texto. ${ }^{63}$ Esto no significa

${ }^{61} Y$ esto puede aplicarse también a su divino Inspirador. Como señala una comentarista, "Dios no se ha puesto a sí mismo a prueba en la Biblia por medio de palabras, de lógica, de retórica". Elena de White, Mensajes selectos (Mountain View, CA: Publicaciones Interamericanas, 1966), 1:24.

${ }^{62}$ Es necesario tener en cuenta que lo que actualmente se denomina el método histórico-gramatical se desarrolla principalmente a partir de la Reforma y con posterioridad a ella. Véase al respecto Raymond F. Surburg, "The Presuppositions of the Historical-Grammatical Method as Employed by Historic Lutheranism", The Springfielder 38:4 (1974) 278-288. El método pretende ser un abordaje científico a la vez que respetuoso del texto bíblico pero en parte se desarrolla luego de la Reforma en reacción a los enfoques de estudio bíblico surgidos a partir de los siglos XVII y XVIII usualmente conocidos como alta crítica o método histórico-crítico.

${ }^{63}$ Qué es lo que tradicionalmente el método histórico-gramatical se ha propuesto. Ver por ejemplo Samuel Koranteng-Pipim, Recibiendo 
que los autores del NT alegorizaran las Escrituras del Antiguo sino que hacían de ella una lectura de carácter más teológico que exegético. Parece claro que los autores del NT hacen a menudo una lectura cristológica, eclesiológica y tipológica de los textos del Antiguo. ${ }^{64}$ Pero todas éstas son categorías teológicas y apuntan a una comprensión del texto que va más allá de la "letra" o la superficie del mismo; se trata de una comprensión que apunta a entender la Biblia como un todo y donde sin duda el AT completo y la revelación posterior de Dios en Cristo (que da origen al NT) se constituyen en el contexto total de interpretación de los autores del NT. No se trata sólo de que los textos del AT son interpretados a la luz del contexto del Nuevo. El contexto es la revelación completa. Y esto sitúa a los autores de NT en un papel

la Palabra. ¿Cómo afectan a nuestra fe los enfoques bíblicos?, trad. David P. Gullón (Buenos Aires: Asociación Casa Editora Sudamericana, 1996), 415, quien considera que el método histórico gramatical busca "interpretar las Escrituras según su sentido sencillo, literal, evidente, directo o corriente". Una metodología como ésta sin duda se encuentra intrínsecamente limitada para descubrir y comprender un posible sentido más completo en el texto. “Casi por definición, los métodos históricos-gramaticales y críticostradicionales de exégesis no pueden detectar o comprender tal sentido más completo [sensus plenior]". William W. Klein, Craig L. Blomberg y Robert L. Hubbard, Jr. Introduction to Biblical Interpretation, ed. revisada y actualizada (Nashville, TN, 2004), 178. Tal vez por ello algunos eruditos bíblicos contemporáneos conservadores prefieren hablar de "método teológicohistórico", Hasel, La interpretación de la Biblia, 93; "crítica históricoteólógica", George Eldon Ladd, Crítica del Nuevo Testamento: Una perspectiva evangélica, trad. Moisés Chávez (El Paso, TX: Editorial Mundo Hispano), 33; o "método histórico-bíblico", Richard M. Davidson, "Interpretación bíblica", en Raoul Dederen, ed., Tratado de Teología Adventista del Séptimo Día trad. David P. Gullón, et al. (Buenos Aires: Asociación casa Editora Sudamericana, 2009), 109.

${ }^{64}$ Ver LaRondelle, The Israel of God in Prophecy, 35-123. 
que no es el de meros intérpretes o exégetas que buscan comprender el sentido liso y llano de un texto, sino en un papel más cercano al de teólogos (cuya tarea va más allá del trabajo exegético). ${ }^{65}$ Esto a su vez implica que no se puede comprender la forma en que el NT interpreta el Antiguo en términos de su similitud con la metodología de la teología bíblica y la exégesis contemporánea. ${ }^{66}$

En síntesis, la investigación del uso del AT en el NT debe permitir que sean los propios autores bíblicos los que provean las presuposiciones teológicas, hermenéuticas y metodológicas que conduzcan a una comprensión cabal de la forma en que el AT es entendido por el Nuevo. De esta tarea puede emerger un modelo bíblico más comprensivo que conduzca al intérprete contemporáneo en su aproximación a las Escrituras Sagradas.

${ }^{65} \mathrm{~A}$ título de ejemplo puede ser de utilidad analizar la forma en que los autores del NT utilizan y conectan entre sí textos del AT en pasajes tales como Rom 3:10-18; 4:3-24; Gál 3:6-18; Heb 1:5-13; Sant 2:21-25; etc.

${ }^{66}$ Algunos eruditos del área de la teología sistemática incluso ven en el uso de la Biblia Hebrea por parte de los autores neotestamentarios un uso más próximo a la interpretación que la sistemática hace de la Biblia que el que propone la teología bíblica o la exégesis. Frank Hasel ha señalado que "la Escritura misma contiene formas de presentaciones, discusiones, $\mathrm{y}$ argumentos que son compatibles con una elaboración [teológica] 'sistemática'". Frank Hasel, "Algunas reflexiones sobre la relación entre la Teología Sistemática y Teología Bíblica”, Theologika 11:1 (1996) 120. Hasel sigue en este punto a Tomas N. Figuer, "Biblical and Systematic Theology Interaction: A Study on the Atonement", en Ben C. Ollenburger, ed., So Wide a Sea: Essays on Biblical and Systematic Theology (Elkart: Institute of Mennonite Studies, 1991), 3-5. 